\title{
ON THE TWO-VARIABLE CONWAY POTENTIAL FUNCTION
}

\author{
MARK E. KIDWELL
}

\begin{abstract}
The Conway potential function $\nabla(r, s)$ of a link with one unknotted component labeled $s$ and all other components labeled $r$ can be computed recursively using the first two Conway identities. $\nabla(r, s)$ can be written uniquely as a polynomial in $z_{1}=r-r^{-1}, z_{2}=s-s^{-1}$, and the first power of $z_{12}=r s+r^{-1} s^{-1}$.
\end{abstract}

Conway's fundamental announcement $[\mathbf{C}]$ has led to considerable work [B-M, $\mathbf{G}, \mathbf{H}, \mathbf{K}_{1}, \mathbf{K}_{2}$ ] on the potential function of a knot or link. Most of this work except $[\mathbf{H}]$ has dealt with the one-variable potential function $\nabla(z)$, a normalized and specialized form of the reduced Alexander polynomial. An attractive feature of $\nabla(z)$ is that it may be computed recursively using Conway's first identity (1.1) and the fact that $\nabla(z)=1 / z$ for the unknot. According to [B-M], $\nabla(z)$ may even be defined recursively.

Conway's first identity (a result actually known to Alexander [A, pp. 301-302]) cannot be applied to a crossing of a link projection in which the two strands have distinct labels. Distinct labels lead to a potential function with more than one variable. Hartley $[\mathbf{H}]$ asks whether the several-variable potential function can be computed recursively and gives a positive answer for two-bridge links. Kauffman $\left[\mathbf{K}_{2}\right.$, p. 111] also asks how may "generators of the polychrome skein" are required to compute the several-variable potential function recursively.

In this paper, we show how to compute recursively the two-variable potential function $\nabla(r, s)$ of a link having one component unknotted and labeled $s$ and all other components labeled $r$. Since a two-bridge link consists of two unknotted components, our result generalizes that of Hartley. To start, we must know the potential functions of the unknot, split links, and Hopf links.

Our main tool is Conway's second identity (1.2(a), (b)), which can be used on tangles with two distinct labels and which respects the integrity of link components as Conway's first identity does not. We shall also need the formula for the potential function of the connected sum of two links. Conway's first identity will do its share of the work in simplifying the $r$-components of the link.

This paper is written with the expectation that a Jones-type polynomial $[\mathbf{F}$, $\mathbf{J}] P\left(x, y, z_{1}, z_{2}, \ldots\right)$ which distinguishes individual link components will soon be defined. If, as seems likely, $P\left(x, y, z_{1}, z_{2}, \ldots\right)$ satisfies a linear identity involving the same three links as Conway's second identity, the arguments of this paper will hold equally well for it.

Hugh Morton $[\mathbf{M}]$, while proving a reversal result for the Jones polynomial, uses an argument quite similar to ours in its sequence of steps. Our efforts were completely independent.

Received by the editors August 28, 1985.

1980 Mathematics Subject Classification (1985 Revision). Primary 57M25; Secondary 39A10.

Key words and phrases. Conway potential function, good link, brace notation. 
It has become popular in papers dealing with the one-variable Conway potential function to replace the variable $\{t\}=t-t^{-1}$ with $z$. In $\S 2$, we indicate the somewhat more complicated procedure required to put the two-variable potential function in "positive exponent" form.

1. Recursive computation of the potential function. In this paper, a knot is regarded as a special kind of link. Following Conway and Hartley, we consider the potential function of a knot to have the form

$$
\left(a_{0}+a_{1}\left(t^{2}+t^{-2}\right)+\cdots+a_{n}\left(t^{2 n}+t^{-2 n}\right)\right) /\left(t-t^{-1}\right) .
$$

We consider links to be embedded in $\mathbf{R}^{3}$. As in $[\mathbf{C}]$, each component of a link carries a (not necessarily distinct) label.

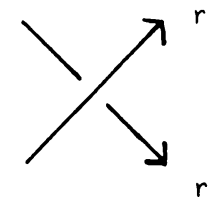

(a) $L: I_{j}=1$

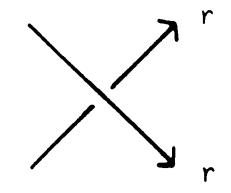

(b) $S_{j} L: I_{j}=-1$

FIGURE 1

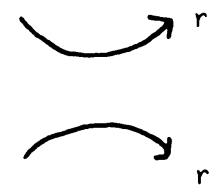

(c) $E_{j} L$

In order to apply Conway's identities, we must consider regular projections $P(L)$ of the link $L$ onto a plane. The crossings (double points) of these projections will be called pure if both strands of the crossing have the same label and mixed if they have distinct labels.

Conway's first identity involves a given link $L$ and two links $S_{j} L$ and $E_{j} L$ (the $j$ th surgery and elimination, in the language of $[\mathbf{B}-\mathbf{M}]$ ) which differ from $L$ only in a neighborhood of a pure crossing $V_{j}$ in $P(L)$. The relevant portions of the three links are displayed in Figure 1. Let $I_{j}$ be a crossing index \pm 1 which is opposite for $L$ and $S_{j} L$. The first identity relates the potential functions of these three links as

$$
\nabla(L)-\nabla\left(S_{j} L\right)=I_{j}(L)\left(r-r^{-1}\right) \nabla\left(E_{j} L\right) .
$$

Most proofs of this identity have been given for the one-variable potential function, but they apply equally well to the several-variable case provided $V_{j}$ is a pure crossing. In at least one of the three links, one inevitably has two distinct components with the same label, however.

Conway's second identity may be applied when a link projection $P(L)$ has a region $R_{k}$ in its plane complement bounded by two edges and two mixed crossings of the same index. Such a configuration will be called a clasp. There are two cases, illustrated in Figures 2(a) and 2(d), depending on whether the boundary of $R_{k}$ is consistently oriented. The double surgery $S_{k k}$ switches the index of both crossings. The double elimination $E_{k k}$ gets rid of both crossings as shown in Figures 2(c) and 2(f). In the case of Figures 2(a)-(c),

$$
\nabla(L)+\nabla\left(S_{k k} L\right)=\left(r s+r^{-1} s^{-1}\right) \nabla\left(E_{k k} L\right) .
$$

In the case of Figures 2(d)-(f),

$$
\nabla(L)+\nabla\left(S_{k k} L\right)=\left(r s^{-1}+r^{-1} s\right) \nabla\left(E_{k k} L\right) .
$$




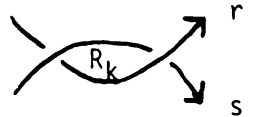

(a) $\mathrm{L}$

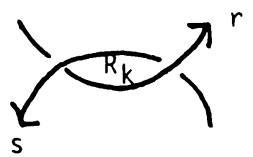

(d) $\mathrm{L}$

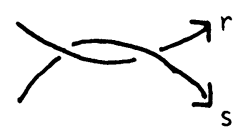

(b) $\mathrm{S}_{k \mathrm{k}} \mathrm{L}$

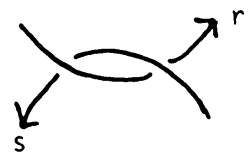

(e) $\mathrm{S}_{k \mathrm{k}} \mathrm{L}$

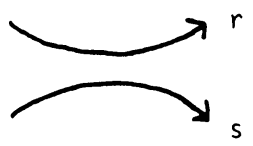

(c) $E_{k k} L$

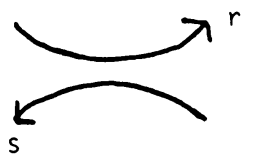

(f) $E_{k k} L$

FIGURE 2

Conway's second identity may also be used when the crossings of a clasp are pure (simply identify $r$ and $s$ ), but it is then a simple consequence of the first identity.

The following recursive definition is meant to capture the notion that $L$ can be reduced to the simplest links by a sequence of single and double surgeries and eliminations and by factoring connected sums.

DEFINITION 1. A link $L$ in $\mathbf{R}^{3}$ is good if it meets at least one of the following criteria:

(a) The unknot, all split links, and the Hopf links (the simplest links of linking number \pm 1 ) are good. (They may be labeled arbitrarily.)

(b) The connected sum of two good links is good.

(c) If $L$ has a projection $P$ with a pure crossing $V_{j}$ such that $S_{j} L$ and $E_{j} L$ are good, then $L$ is good.

(d) If $L$ has a projection $P$ with a clasp $R_{k}$ such that $S_{k k} L$ and $E_{k k} L$ are good, then $L$ is good.

Definition 1(a) implies that any link having a projection with at most two crossings is good. The potential function of the unknot is $1 /\left(t-t^{-1}\right)$, the potential function of any split link is 0 , and the potential function of the Hopf links is \pm 1 (the same as their linking number). If the two factors $L_{1}$ and $L_{2}$ of a connected sum are joined together along a component labeled $r$, then

$$
\nabla(L)=\nabla\left(L_{1}\right)\left(r-r^{-1}\right) \nabla\left(L_{2}\right)
$$

[C, p. 338]. As indicated by Conway and worked out in detail in [B-M and $\mathbf{K}_{2}$ ], any link in which all components have the same label is good.

For the rest of this section, we assume that our link $L$ has an unknotted component $L_{s}$ labeled $s$. All other components are labeled $r$ and are known collectively as $L_{r}$. We assume that $L_{s}$ is a geometric circle in the plane $\mathbf{R}^{2} \times\{0\}$. All our projections will be regular projections onto that plane. Thus $P\left(L_{s}\right)=L_{s}$. The two open regions of $\mathbf{R}^{2} \times\{0\}-L_{s}$ will be called Int and Ext in the obvious fashion. All our projections will be drawn from the positive- $z$ direction, and $L_{s}$ will be oriented clockwise in them.

LEMMA 1. There is a projection $P$ of $L$ with $P\left(L_{r}\right) \cap$ Int consisting of a union of disjoint line segments, each undercrossing $L_{s}$ at one end and overcrossing at the other. 


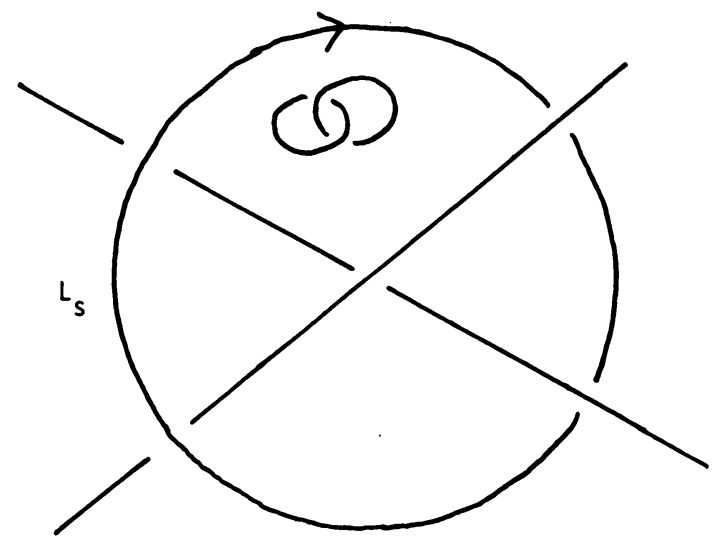

FIGURE 3
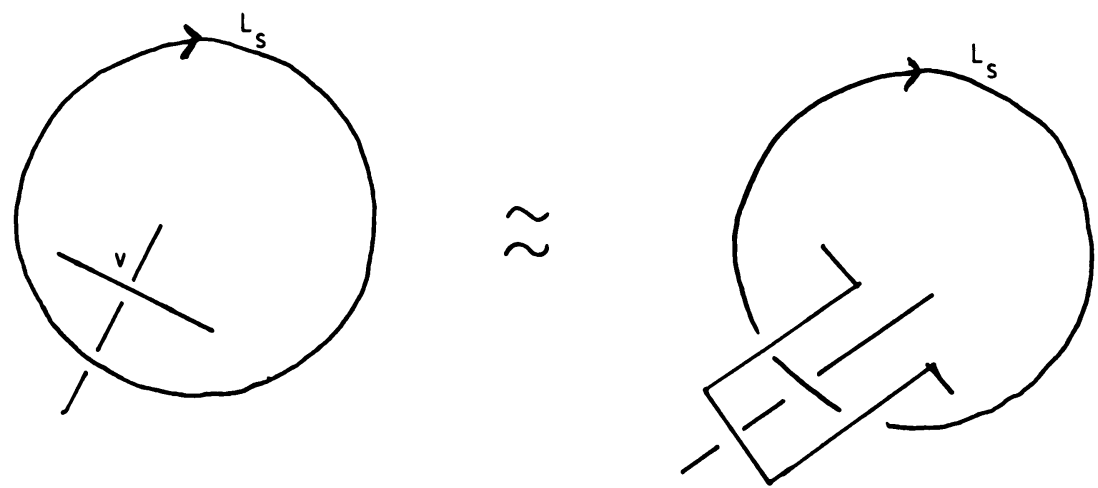

FIGURE 4(a)
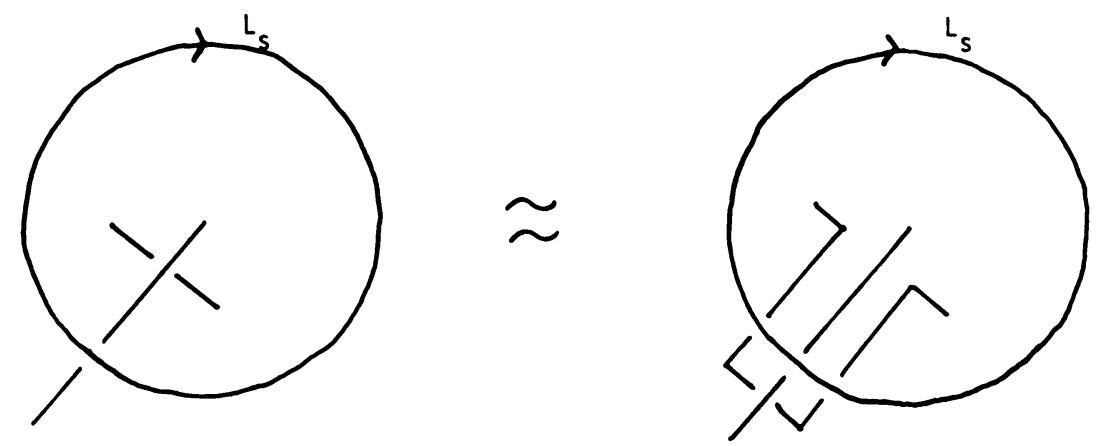

FIGURE 4(b)

Proof. Consider the planar graph $L_{s} \cup\left(P\left(L_{r}\right) \cap\right.$ Int). If this graph is not connected (Figure 3), remove any "islands" from Int to Ext. If there are any vertices remaining in Int, they are connected to $L_{s}$ by a path in the graph, and at least one vertex is only a single edge away from $L_{s}$. This vertex $v$ can be removed from Int as shown in Figure 4(a) or (b). Repeat this procedure until all internal vertices have been removed. If any of the remaining segments of $P\left(L_{r}\right) \cap$ Int overcross or undercross $L_{s}$ at each end, they may be removed from Int as shown in Figure 5. 

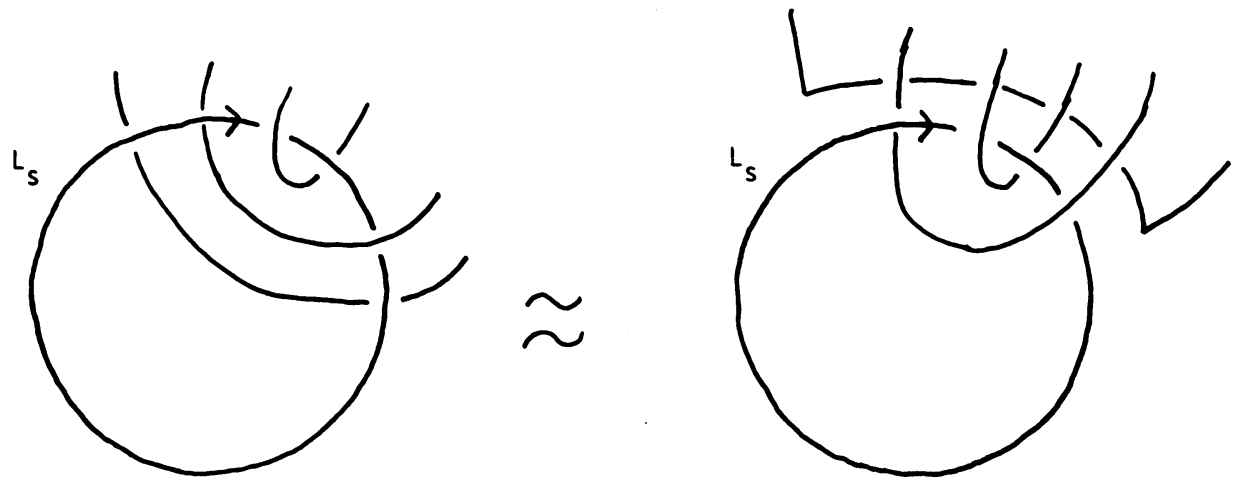

FIGURE 5
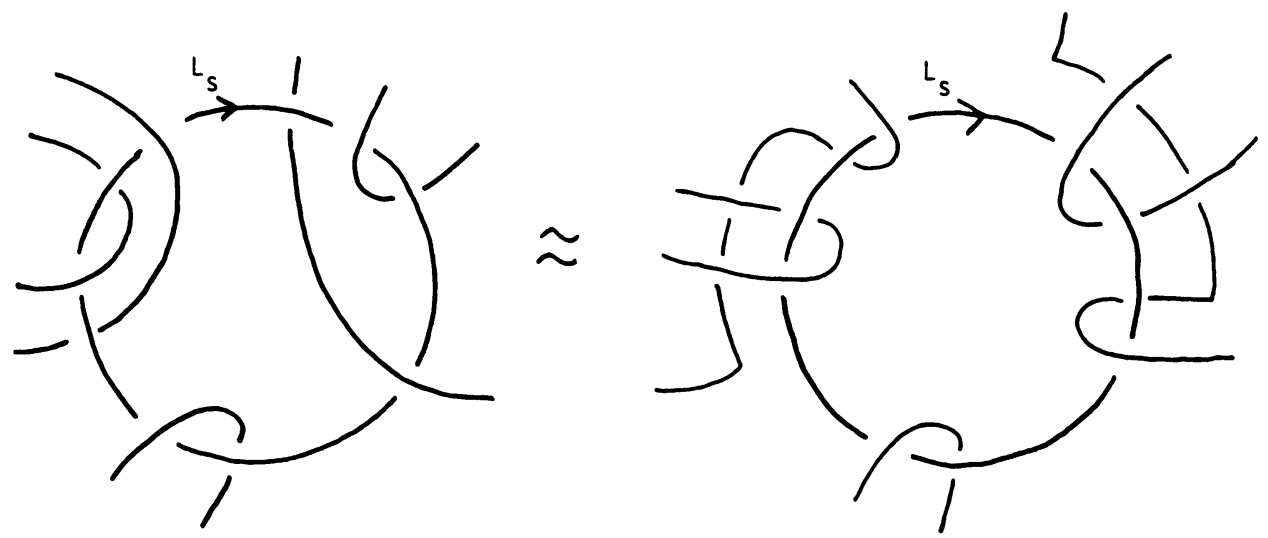

FIGURE 6
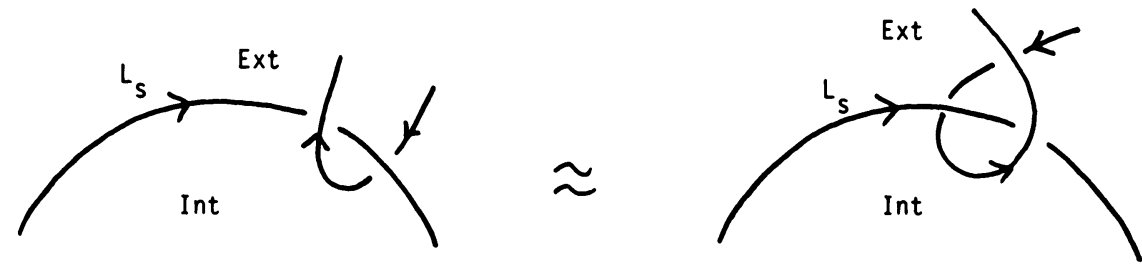

FIGURE 7

Each of the remaining segments in $P\left(L_{r}\right) \cap$ Int divides Int into two subdisks. If one of these subdisks contains no other $r$-segments, then there is a clasp with boundary edges the given segment of $P\left(L_{r}\right)$ and a segment of $L_{s}$. There must be at least two such clasps in any projection satisfying Lemma 1 , unless $P\left(L_{r}\right) \cap \operatorname{Int}=\varnothing$.

LEMMA 2. There is a projection of $L$ satisfying Lemma 1 in which every segment of $P\left(L_{r}\right) \cap$ Int is part of the boundary of a clasp.

PROOF. The technique for changing a nonclasping segment to a clasping segment is shown in Figure 6. The property of undercrossing $L_{s}$ at one end and overcrossing at the other is preserved. 


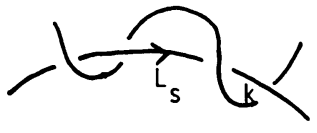

(a) $\mathrm{L}$

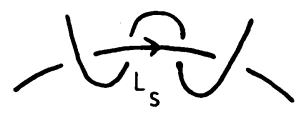

(b) $\mathrm{S}_{k k} \mathrm{~L}$

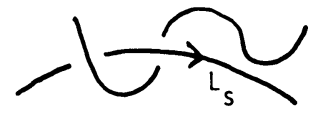

(c) $E_{k k} \mathrm{~L}$

FIGURE 8

Let there be $n$ clasps in the projection of $L$ that we have now reached. If $n \geq 2$, $L_{s} \cup\left(P\left(L_{r}\right) \cap\right.$ Int) consists of one $2 n$-sided "middle" as well as the $n$ clasps. Figure 7 shows that the orientation of a segment of $P\left(L_{r}\right) \cap$ Int can be reversed without otherwise changing the projection inside Int. Henceforth we will assume that all edges around the "middle" are oriented clockwise. An arbitrary clasp is labeled number 1 , and its two crossings labeled $1_{i}$ (for incoming) and $1_{o}$ (for outgoing). The crossing points along $L_{s}$ are then labeled, in clockwise order, $1_{\mathrm{i}}, 1_{\mathrm{o}}, 2_{\mathrm{i}}, 2_{\mathrm{o}}, \ldots, n_{\mathrm{i}}, n_{\mathrm{o}}$.

Let us call a projection of $L$ clasped if it satisfies all the conditions that we have proved possible thus far. Among all clasped projections of $L$, select one in which

(a) the number $n(L)$ of clasps is minimal,

(b) the number $m(L)$ of pure (r-over- $r$ ) crossings is minimal, consistent with (a).

LEMMA 3. If $n(L)=0$ or 1 , then $L$ is a good link.

PROOF. If $n(L)=0$, then $L$ is a split link, hence good by Definition 1(a). If $n(L)=1$, then $L$ is the connected sum of a Hopf link and a link with all labels $r$. Since both of its factors are good, $L$ is good by Definition $1(\mathrm{~b})$.

LEMMA 4. If $n(L) \geq 2$ and $m(L)=0$, then $L$ is a good link.

PROOF. By Lemma 3 and induction, assume that all links with clasp number smaller than $n(L)$ are good. Since $m(L)=0$, Ext resembles Int after Lemma 1 was applied but before Lemma 2 was applied. As observed there, Ext contains at least two clasps. Figure $8(\mathrm{a})$ shows one of these exterior clasps together with its two neighboring interior clasps. (The situation must be as in Figure 8(a) or its mirror image, since we are assuming that $n(L)$ is minimal.) If $k$ is one of the pictured clasps, then the links $S_{k k} L$ and $E_{k k} L$ of Figures $8(\mathrm{~b})$ and (c) have smaller clasp numbers than $L$, hence are good. By Definition $1(\mathrm{~d}), L$ is also good.

THEOREM 1. Any link $L$ with one unknotted component labeled $s$ and all other components labeled $r$ is good.

Proof. Assume $L$ has been given a clasped projection with minimal $n(L)$ and $m(L)$. By Lemmas 3 and 4 and induction, assume that any such link with smaller $n$ or smaller $m$ and equal $n$ is good.

Let $k$ be the segment of $L_{r}$ that originates at $1_{o}$ and terminates when it reenters Int. Let this reentry crossing be called $j_{\mathrm{i}}$. Note that $j_{\mathrm{i}}=1_{\mathrm{i}}$ is possible. Let the pure crossings along $k$ in which only one segment is part of $k$ be labeled in order $k_{1}, \ldots, k_{r}$. Figure $9(\mathrm{a})$ shows a typical situation. (The reader is to imagine that $m(L)$ is minimal.)

As in $[\mathbf{B}-\mathbf{M}]$, we now perform a surgery and elimination at any crossing $k_{\mathrm{i}}$ in which $k$ is the overcrossing segment. (These crossings are circled in Figure 9(a).) By induction on $m(L)$, the links produced by elimination during this process will all be good. Let $S L$ be the link obtained by performing surgeries at all the necessary 


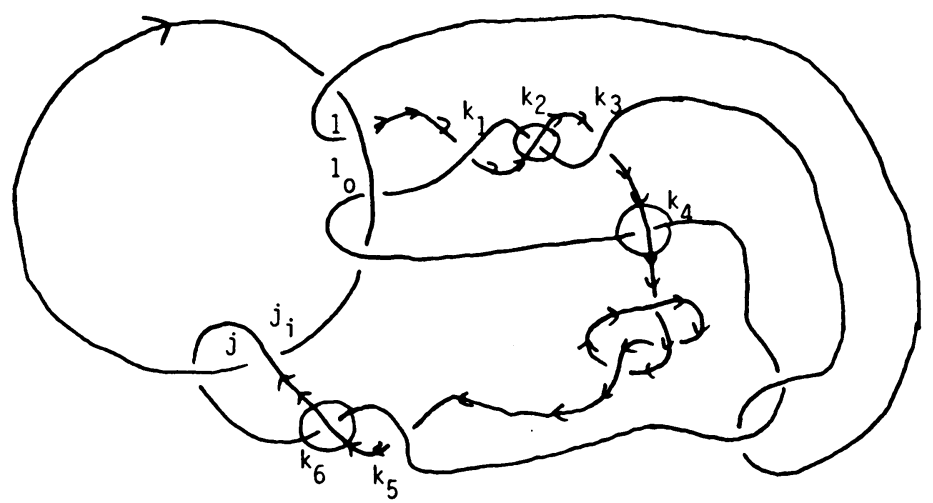

FIGURE 9(a)

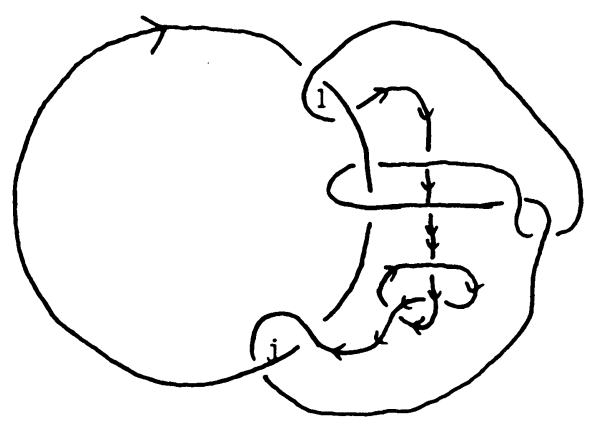

FIGURE 9(b)

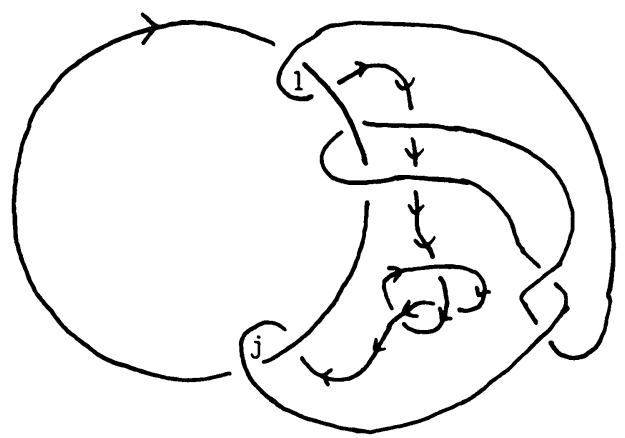

Figure 9(c)

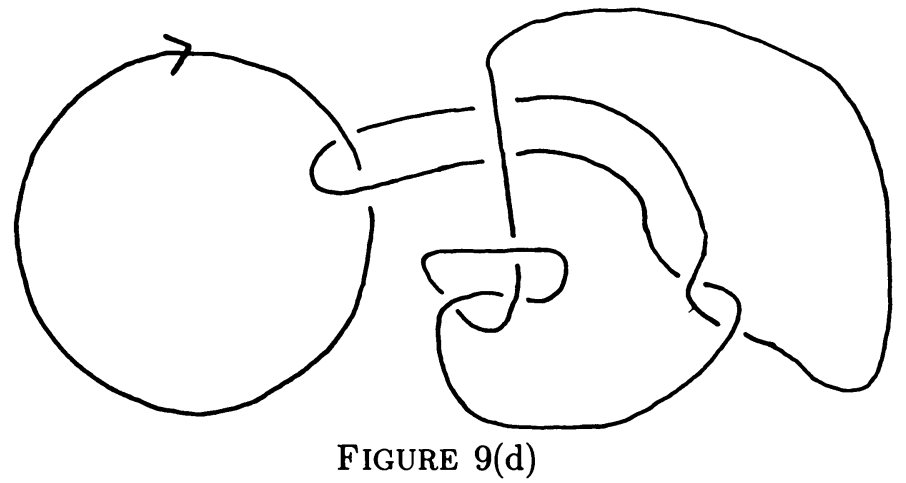

crossings. By possibly multiple application of Definition $1(\mathrm{c}), L$ will be good if and only if $S L$ is good. Let $S k$ be the segment of $S L$ corresponding to segment $k$ of $L$. By construction, $S k$ lies below the rest of $S L_{r}$, and so may be moved to the position of Figure $9(\mathrm{~b})$.

Assume for the moment that $j_{\mathrm{i}} \neq 1_{\mathrm{i}}$. By double surgeries and eliminations on clasps 1 and $j$ as necessary, we obtain a link $S^{\prime \prime} L$ in which the segment $S^{\prime \prime} k$ corresponding to $S k$ undercrosses at both $1_{\mathrm{o}}$ and $j_{\mathrm{i}}$ (see Figure $9(\mathrm{c})$ ). The links obtained by double elimination are good since they have smaller clasp numbers than $L$. By Definition 1(d) (applied zero, one or two times), $S L$ is good if and only 

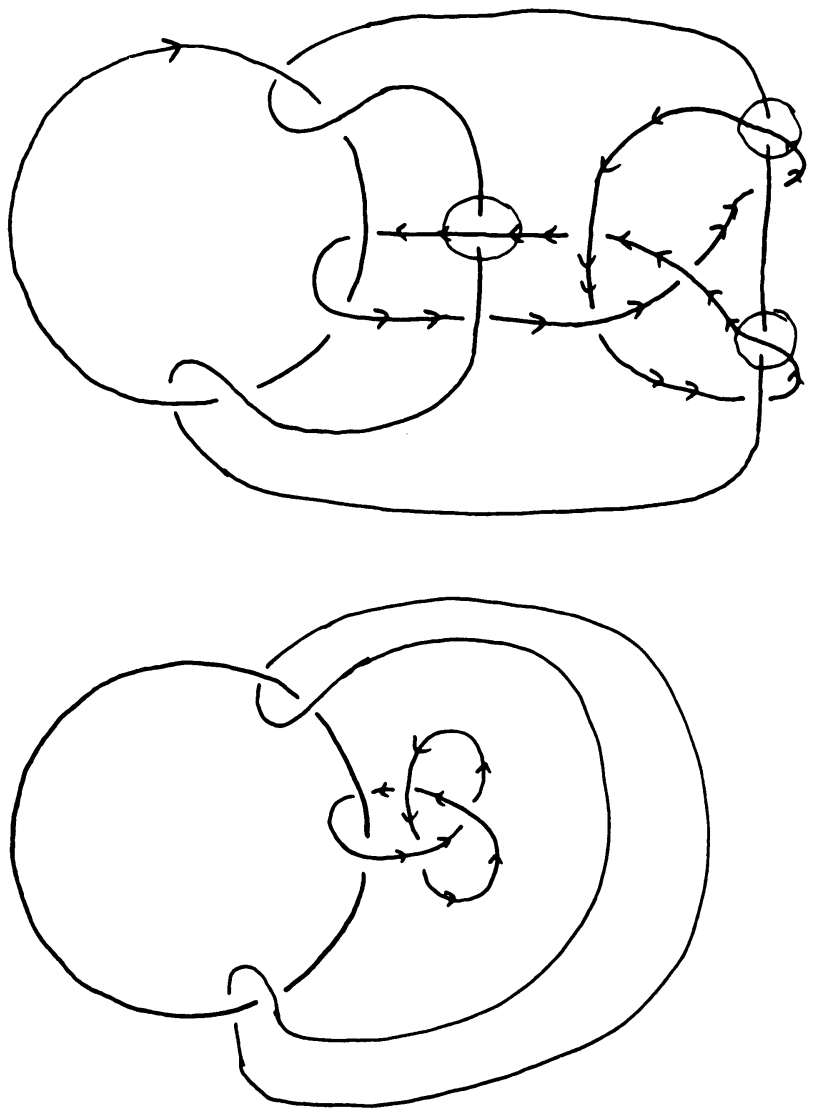

FIGURE 10

if $S^{\prime \prime} L$ is good. But as shown in Figure 9(d), $S^{\prime \prime} L$ has clasp number less than or equal to $n(L)-2$, hence it is good by induction. This is sufficient to make $L$ good.

Now assume that $\mathbf{1}_{\mathrm{i}}=j_{\mathrm{i}}$. In this case our single surgery procedure produces a link that is a connected sum of a link with clasp number 1 and a link with clasp number at most $n(L)-1$. Since both factors are good, $S(L)$ and hence $L$ are good (see Figure 10).

2. A notation for the two-variable potential function. According to Conway's brace notation, $\{f(r, s)\} \equiv f(r, s)+f\left(-r^{-1},-s^{-1}\right)$ where $f$ is a two-variable Laurent polynomial. Use of the brace notation requires care, as the following observations indicate.

(a) $\left\{r^{n}\right\} \neq\{r\}^{n}$ for $n>1$ and $\{r s\} \neq\{r\}\{s\}$.

(b) If 1 is substituted for $s$ in $\{f(r, s)\}$, the result is not necessarily $\{f(r, 1)\}$. For example, substituting 1 for $s$ in $\{r s\}=r s+r^{-1} s^{-1}$ yields $r+r^{-1}$, which cannot be written in brace notation.

(c) $\left\{r^{-n}\right\}=(-1)^{n}\left\{r^{n}\right\}$ and $\left\{r^{n} s^{-n}\right\}=\left\{r^{-n} s^{n}\right\}$.

According to Conway and Hartley, the potential function $\nabla(r, s)$ is the polynomial $\Delta\left(r^{2}, s^{2}\right)$ multiplied by a Laurent monomial $r^{\mu_{1}} s^{\mu_{2}}$. Thus the parity of the $r$-degree and the $s$-degree of each term in $\nabla(r, s)$ is the same. The parity of the total degree of each term in $\nabla(r, s)$ and of the degree in each term of $\nabla(r, r)$ is also the 
same. Hartley [H, Theorem 4.7] proves that if $c \geq 2$ is the number of components of the link, then the degree of each term of $\nabla(r, r)$ is even if $c$ is even and odd if $c$ is odd. The same then applies to the total degree of each term in $\nabla(r, s)$.

The main symmetry property of the potential function $[\mathbf{H}$, Theorem 5.5] is that

$$
\nabla(r, s)=(-1)^{c} \nabla\left(r^{-1}, s^{-1}\right) .
$$

From this and the previous paragraph, we have that if $c$ is even and the coefficient of $r^{m} s^{n}$ in $\nabla(r, s)$ is $A \neq 0$, then $m+n$ is even and the coefficient of $r^{-m} s^{-n}=$ $(-r)^{-m}(-s)^{-n}$ will also be $A$. If $C$ is odd and the coefficient of $r^{m} s^{n}$ is $A \neq 0$, then $m+n$ is odd and the coefficient of $r^{-m} s^{-n}=-(-r)^{-m}(-s)^{-n}$ will be $-A$. In either case, the sum of the two symmetrical terms can be written $A\left\{r^{m} s^{n}\right\}$.

By addition, we have that the set of brace symbols $\left\{r^{m} s^{n}\right\}$ generates all twovariable potential functions. To move exponents outside of braces as much as possible, we use two forms of an identity derived from the definition of the brace notation:

$$
\begin{aligned}
& \left\{r^{m+1} s^{n}\right\}=\{r\}\left\{r^{m} s^{n}\right\}+\left\{r^{m-1} s^{n}\right\} \\
& \left\{r^{m} s^{n+1}\right\}=\left\{r^{m} s^{n}\right\}\{s\}+\left\{r^{m} s^{n-1}\right\}
\end{aligned}
$$

This identity bears a striking resemblance to Conway's first identity. The proof is a routine Laurent polynomial manipulation.

The identity (2.2) allows us to reduce (or raise) exponents in $\left\{r^{m} s^{n}\right\}$ until all terms have exponent zero or one. For example,

$$
\left\{r^{-1} s\right\}=\{r s\}-\{r\}\left\{r^{0} s\right\}=\{r s\}-\{r\}\{s\} .
$$

Annoyingly, $\left\{r^{0} s^{0}\right\}=2$, but we can generate all two-variable potential functions from $1,\{r\},\{s\}$, and $\{r s\}$.

All $\left\{r^{m} s^{n}\right\}$ can be written in terms of the sequence of Fibonacci polynomials [B] defined by $F_{0}(x)=0, F_{1}(x)=1$, and $F_{k+1}(x)=x F_{k}(x)+F_{k-1}(x)$. One form of the expression is

$$
\left\{r^{m} s^{n}\right\}=F_{m}(\{r\}) F_{n}(\{s\})\{r s\}+F_{m+1}(\{r\}) F_{n-1}(\{s\})+F_{m-1}(\{r\}) F_{n+1}(\{s\}) .
$$

For $k>0, F_{-k}(x) \equiv(-1)^{k+1} F_{k}(x)$.

We now introduce the notation $\{r\}=z_{1},\{s\}=z_{2}$, and $\{r s\}=z_{12}$.

THEOREM 2. Any two-variable potential function $\nabla(r, s)$ can be written uniquely as

$$
f_{1}\left(z_{1}, z_{2}\right)+z_{12} f_{2}\left(z_{1}, z_{2}\right)
$$

where $f_{1}$ and $f_{2}$ are two-variable polynomials, $z_{1}=\{r\}, z_{2}=\{s\}$, and $z_{12}=\{r s\}$. There are links for which $f_{2}$ is not identically zero.

PROOF. Formula (2.3) shows that $\left\{r^{m} s^{n}\right\}$, and hence any potential function, can be written in the form of (2.4).

For uniqueness, it is enough to show that there is no nontrivial identity

$$
f_{1}\left(z_{1}, z_{2}\right)+z_{12} f_{2}\left(z_{1}, z_{2}\right)=0 \text {. }
$$

Suppose there were. Let $A z_{1}^{m} z_{2}^{n}$ be a nontrivial term of largest $z_{1}$-degree in $f_{2}$ and, among these, of largest $z_{2}$-degree. If $f_{2}$ is written as a Laurent polynomial 
in $r$ and $s$, there will be terms of $r$-degree $m$ ranging from $\pm A r^{m} s^{-n}$ to $A r^{m} s^{n}$. Thus if $z_{12} f\left(z_{1}, z_{2}\right)$ is written as a Laurent polynomial, there will be terms of $r$ degree $m+1$ ranging from $\pm A r^{m+1} s^{-n+1}$ to $A r^{m+1} s^{n+1}$. Such an asymmetrical expression cannot equal $-f_{1}\left(z_{1}, z_{2}\right)$.

A consequence of the previous paragraph is that $\{r s\}=z_{12}$ cannot be written as a polynomial $f\left(z_{1}, z_{2}\right)$. Since Conway's 2-component link "4" [C, p. 344] has potential function $\{r s\}$, there are links for which the $z_{12}$ term cannot be eliminated.

The transformation $r \leftrightarrow r^{-1}$ arises in connection with reversing the orientation of the $r$-string(s). So it is perhaps worth noting that under this transformation, $\{r\}=r-r^{-1}$ is replaced by $r^{-1}-r=-\{r\}$ and $\{r s\}$ is replaced by $\left\{r^{-1} s\right\}=\{r s\}-\{r\}\{s\}$. Thus $f_{1}\left(z_{1}, z_{2}\right)+z_{12} f_{2}\left(z_{1}, z_{2}\right)$ is replaced by $\left(f_{1}\left(-z_{1}, z_{2}\right)-\right.$ $\left.z_{1} z_{2} f_{2}\left(-z_{1}, z_{2}\right)\right)+z_{12} f_{2}\left(-z_{1}, z_{2}\right)$.

\section{BIBLIOGRAPHY}

[A] J. W. Alexander, Topological invariants of knots and links, Trans. Amer. Math. Soc. 30 (1928), 275-306.

[B] M. Bicknell, A primer for the Fibonacci numbers: Part VII, Fibonacci Quart. 8 (1970), 407-420.

[B-M] R. Ball and M. L. Mehta, Sequence of invariants for knots and links, J. Physique 42 (1981), 1193-1199.

[C] J. H. Conway, An enumeration of knots and links and some of their algebraic properties, Computational Problems in Abstract Algebra (J. Leech, ed.), Pergamon Press, New York, 1969, pp. 329-358.

[F] P. Freyd, D. Yetter, J. Hoste, W. B. R. Lickorish, K. Millett, and A. Ocneanu, A new polynomial invariant of knots and links, Bull. Amer. Math. Soc. 12 (1985), 239-246.

[G] C. Giller, A family of links and the Conway calculus, Trans. Amer. Math. Soc. 270 (1982), 75-109.

[H] R. Hartley, The Conway potential function for links, Comment. Math. Helv. 58 (1983), 365-378.

[J] V. F. R. Jones, A polynomial invariant for knots via von Neumann algebras, Bull. Amer. Math. Soc. (N.S.) 12 (1985), 103-111.

[K $\left.\mathbf{K}_{1}\right]$ L. Kauffman, The Conway polynomial, Topology 20 (1981), 101-108.

$\left[\mathbf{K}_{2}\right]$ _ Formal knot theory, Mathematical Notes, no. 30, Princeton Univ. Press, Princeton, N. J., 1983.

[M] H. R. Morton, The Jones polynomial for unoriented links, Quart. J. Math. Oxford Ser. 37 (1986), 55-60.

Department of Mathematics, U.S. Naval ACademy, Annapolis, Maryland 21402 